\title{
ON BRIDGING A MODELING SCALE GAP Mesoscale to Microscale Coupling for Wind Energy
}

\author{
Sue Ellen Haupt, Branko Kosovic, William Shaw, Larry K. Berg, \\ Matthew Churchfield, Joel Cline, Caroline Draxl, Brandon Ennis, Eunmo Koo, \\ Rao Kotamarthi, Laura Mazzaro, Jeffrey Mirocha, Patrick Moriarty, \\ Domingo Muñoz-Esparza, Eliot Quon, Raj K. Ral, Michael Robinson, and Gokhan Sever
}

This work has advanced coupled mesoscale to microscale modeling through the terra incognita, generating turbulence at the microscale, testing coupling techniques, and assessing results relevant for wind energy applications.

AfFiliations: Haupt, Kosovic, and Muñoz-Esparza—National Center for Atmospheric Research, Boulder, Colorado; SHAW, BERG, AND RAI-Pacific Northwest National Laboratory, Redmond, Washington; ChURChFIeld, Draxl, Moriarty, Quon, AND RoBINSON-National Renewable Energy Laboratory, Golden, Colorado; CLINE-National Oceanic and Atmospheric Administration, Washington, D.C.; ENNIS-Sandia National Laboratories, Albuquerque, New Mexico; Koo, ANd Mazzaro-Los Alamos National Laboratory, Los Alamos, New Mexico; KotAMARTHI AND SEVER-Argonne National Laboratory, Argonne, Illinois; MIROCHALawrence Livermore National Laboratory, Livermore, California CORRESPONDING AUTHOR: Sue Ellen Haupt, haupt@ucar.edu

The abstract for this article can be found in this issue, following the table of contents.

DOI:10.1175/BAMS-D-18-0033.I

In final form 31 July 2019

C2019 American Meteorological Society

For information regarding reuse of this content and general copyright information, consult the AMS Copyright Policy. most interest occur right in that region, termed the terra incognita (Wyngaard 2004), including siting and operation of wind plants. Thus, to accurately model wind plants, one must devise coupling techniques where the finescale models respond to, or "follow," the changes captured by the detailed atmospheric dynamics and physics of mesoscale models.

Modeling for the wind industry has become increasingly important as the installed capacity of wind energy has grown. The wind industry has grown by a factor of 4.6 in the United States over the past decade (Weissman et al. 2018), with installed capacity exceeding $90 \mathrm{GW}$ in 2018 (American Wind Energy Association 2018). This deployment of wind, together with the advances in solar energy, has changed the paradigm of the energy industry. The fuel for such plants no longer depends on mining fossil fuels, but rather relies on renewable resources. The dynamic nature of atmospheric flow drives the energy transfers between scales that enable harvesting wind energy. Renewable energy generation is challenged by the inherent variability of the atmosphere. Modeling wind plants accurately depends on understanding 
and simulating the ever-changing atmosphere. As a result, meteorologists have engaged with this industry to model this variable energy resource. This modeling enables optimization of wind plants. To accomplish that optimization requires bridging from the mesoscale to the microscale, which are traditionally modeled using differing approaches.

Because the atmosphere drives the changes at the wind plant scale, it is critical to model this variability at the mesoscale. Some of these variations occur regularly, such as those due to the diurnal and seasonal cycles. Others are more episodic, including the large-scale baroclinic weather systems that cause rapid changes in wind speed and direction, or the smaller-scale variations due to more local terrain or land-water characteristics.

Mesoscale models capture the dynamic processes of the atmosphere by including appropriate initial and boundary conditions derived from global models as well as modeling the full range of physics, including radiative transfer, cloud processes, boundary layer processes, interaction with the surface, and other important processes that are seldom included to this extent in microscale models. Additionally, the mesoscale models are nonhydrostatic and include moist processes, which are seldom true for stand-alone microscale models.

Microscale models, on the other hand, are capable of simulating details of flow around terrain, resolving finescale turbulence and explicitly representing interactions with structures such as wind turbines. These microscale large-eddy simulation (LES) models are required to develop new strategies for operating wind plants, such as determining optimal control strategies (Fleming et al. 2014, 2015). They seldom, however, model the full processes of the atmosphere that drive the weather and atmospheric features (moist processes, direct radiative transfer, land surface processes, cloud physics, etc.). Those effects can be driven by coupling the mesoscale models to the LES models.

Mesoscale-microscale coupling presents the most promising approach to addressing the key limitations of current wind plant simulation techniques by combining the advantages of both types of model. Because of this promise, the Mesoscale to Microscale Coupling (MMC) project was formed within the U.S. Department of Energy's (DOE) Atmosphere to Electrons (A2e) initiative, which aims to gain a better physical understanding of wind plant processes and behavior leading to pathways for wind plant innovation. The MMC project has been developing, testing, and evaluating methods and tools to replace the existing highly idealized or steady-state forcing parameters, periodic lateral boundary conditions, and other simplifications typically employed in wind farm simulation tools using microscale models. The key to these improved methods is dynamic input from mesoscale weather models that can provide important meteorological, topographical, and other environmental drivers of microscale variability. Thus, we focus on cases where the mesoscale model performs relatively well and seek to develop methodologies to use that result to force the microscale LES models.

Thus, the goal of the MMC project is to build new high-performance-computing-based multiscale wind plant simulation tools by coupling a broad range of scales to enable the optimization of wind plants, thereby ensuring the efficient, reliable production and integration of future wind-generated electricity. This capability will allow detailed study of flow characteristics of each complex site, allowing better micrositing of wind turbines as well as optimizing control of the plant. It will enable even finer blade-resolved simulations within the LES model that can lead to better design of wind turbines. It will additionally provide a high-fidelity standard that can be used to develop and validate lower-order models.

The overarching objective of the MMC project is to create, assess, and validate state-of-the-science atmospheric simulation methodologies to incorporate important mesoscale flow characteristics into microscale wind plant simulations. Without such coupling, the plant-scale models will not include the full characteristics of the flow and the transfer of energy between the scales will not be correct. Some challenges that must be addressed in coupling these methods correctly include 1) bridging the so-called terra incognita, that grid resolution between about $100 \mathrm{~m}$ and the boundary layer depth at which numerical artifacts are often difficult to distinguish from physical boundary layer rolls; 2 ) testing appropriate methods of coupling the two scales; 3 ) initializing turbulence at the correct scales and locations in the microscale models; 4) providing appropriate and consistent boundary and initial conditions including in complex terrain; and 5) assessing the results and quantifying the uncertainty of the methods.

Figure 1 diagrams the MMC approach that is applied to address these challenges. Our primary approach to improving MMC uses case studies, which allows testing multiple methods and model configurations for rigorous comparison. The project is grounded in data provided by other DOE A2e field studies and projects and was designed to systematically progress from simulating quasi-steady cases 
Approach to Mesoscale to Microscale Coupling

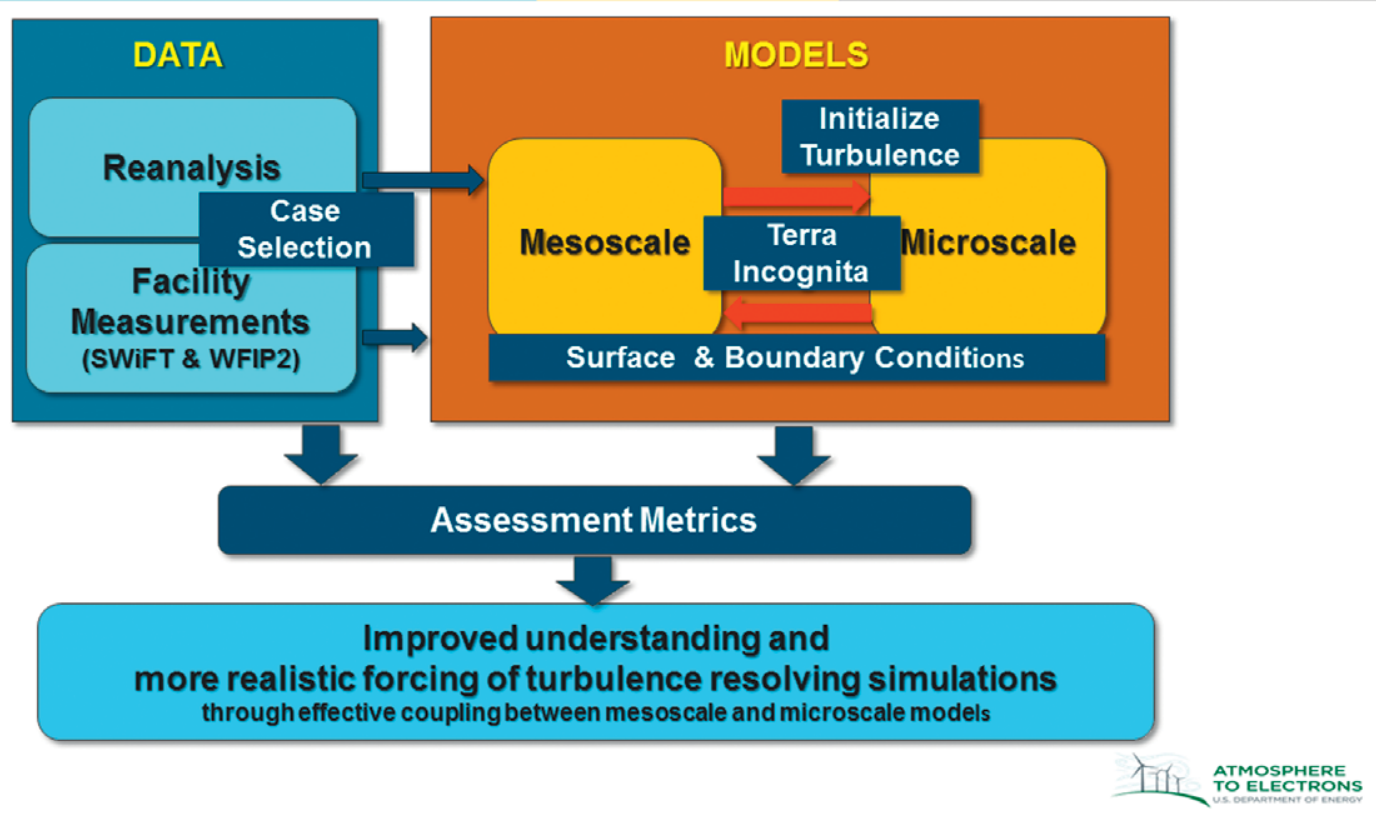

FIG. I. Diagram of the MMC project approach.

through the full complexity of nonstationarity and flow in complex terrain. These data have included measurements taken at the DOE Scaled Wind Farm Technology (SWiFT) facility located at Sandia National Laboratories in Texas, an example of a flat site. Complex terrain cases are studied using data derived from the observations taken in the Pacific Northwest as part of the A2e Wind Forecasting Improvement Project 2 (WFIP 2) described in companion papers (Shaw et al. 2019; Wilczak et al. 2019; Olson et al. 2019; E. Grimit et al. 2019, unpublished manuscript). Including mesoscale forcing in microscale models will also become critical to the success of the A2e Controls Science Project that focuses on innovative wind plant controls (Fleming et al. 2014, 2015). Most prominently, the very specific coupling and modeling philosophies and technologies being developed by the MMC project are necessary for building high-fidelity modeling tools. The project has elicited input from stakeholders in industry and constructed approaches to meet their articulated needs for enabling more efficient plant development and operation.

The remainder of this article details the team's approach to studying those five challenges. The second section describes the models and data that have facilitated this project. Our approach to studying the topics listed above and a sampling of the results appear in the third section. The fourth section provides a discussion of the implications of this work and looks to the remaining work required to facilitate accurate wind plant simulations that could be used to plan new plants, to study methods to optimize turbine control, and to provide other important information to enhance wind plant performance through better knowledge and simulation of the energy-containing processes.

MODELS AND DATA. The MMC project seeks to produce simulations as close to real-world atmospheric conditions as possible; thus, cases are selected from observational studies and modeling setups strive to capture the dominant atmospheric processes present during those times. To that end, the team tested several ways of simulating at both the mesoscale and microscale (Haupt et al. 2015; Mirocha et al. 2018). Here, we focus on the models that have been selected for coupling and on the data used to assess how to address the challenges listed in the first section.

Mesoscale model-WRF. The open-source Weather Research and Forecasting (WRF) Model (Skamarock et al. 2008) is used to represent the mesoscale atmospheric flow. WRF's equations are fully compressible, Euler nonhydrostatic. It uses terrain-following hydrostatic-pressure vertical coordinates with vertical grid stretching permitted.

Atmospheric flow and its energy derive from more than the dynamics: the physical processes provide important forcings and are modeled via parameterizations. One key parameterization involves radiative transfer processes, which drive the 
heat balance throughout the modeled system. The incoming shortwave solar radiation heats the surface and atmospheric constituents. Land surface models quantify land surface properties (including land vs water as well as soil type, temperature, and moisture and vegetation) and their interactions with the atmosphere. These land surface models help determine the ground temperature, which determines the emission of longwave radiation and provides the fluxes of heat, moisture, and momentum to the planetary boundary layer (PBL) parameterization (Stensrud 2007). The PBL schemes determine the vertical mixing of the atmospheric boundary layer (ABL), which accounts for the vertical distribution of heat, water vapor, horizontal momentum, and trace gases. The surface turbulent fluxes are typically modeled via a surfacelayer scheme based on Monin-Obukhov similarity theory (Monin and Obukhov 1954). Shallow and deep convection are modeled via cumulus parameterizations, which determine temperature and water vapor profiles resulting in condensation for cloud formation and precipitation. This balance of water vapor impacts the density profiles, which in turn impacts the energy distribution of the atmosphere. Finally, the microphysics parameterizations determine the formation and distribution of cloud and ice droplets. Because these parameterizations interact with each other, one must be careful to choose compatible sets of parameterizations (Stensrud 2007; Warner 2011).

WRF-LES. WRF allows various configurations and nesting capabilities, including into the LES ranges (Moeng et al. 2007; Mirocha et al. 2010), forming WRF-LES. Thus, one approach to coupled modeling leverages this capability, including the full physics suite within WRF. Mesh refinement is provided via block rectangular nesting, with integer ratios for the horizontal mesh and time stepping ratios. Vertical mesh refinement is available either as an external postprocessing step or can be activated for concurrent simulation (Daniels et al. 2016).

Various wind-energy-specific modules have been previously implemented in WRF, including embedding actuator disk models of wind turbines, which simulate the impact of wind turbines on the momentum and energy of the flow (Mirocha et al. 2014) and parameterizations of wind farms (Fitch et al. 2012). WRF has been applied to wind resource assessment (Fernandez-Gonzalez et al. 2018), wind power forecasting (Cheng et al. 2017; Mahoney et al. 2012), and for modeling the impacts of wind farms on flow in the immediate area and downstream of wind farms (Rajewski et al. 2014; Mirocha et al. 2015).
Stand-alone microscale models. The MMC project has employed the LES model, Simulator for Wind Farm Applications (SOWFA; National Renewable Energy Laboratory 2015), which is a collection of dynamics models, turbulence models, turbine models, boundary conditions, and utilities written specifically for computing wind plant flows. The complete toolset is meant to span from the mesoscale down to the explicit turbine scale, providing interfaces between tools of different scales.

The SOWFA microscale solver is built upon the Open-source Field Operations and Manipulations (OpenFOAM) CFD toolbox (OpenFOAM 2014), a popular, open-source, freely available set of $\mathrm{C}++$ libraries for solving partial differential equations. OpenFOAM comes with a variety of standard flow models, turbulence models, boundary conditions, and other physics models, and because of its open-source nature, it is relatively straightforward to develop new models and boundary conditions. OpenFOAM, and hence SOWFA, use an unstructured-mesh, finite-volume formulation for solving the governing equations. A variety of options exist for spatial discretization, and we typically use second-order central differencing for the advective and diffusive terms. Time discretization is secondorder backward differencing. The solution advances sequentially by solving the equation set using Issa's pressure-implicit splitting operation (Issa 1986) with the ability to employ an outer iteration loop necessary to maintain second-order accuracy in time. SOWFA's microscale flow solver is incompressible, but uses the Boussinesq approximation for buoyancy, and could be readily extended to solve the anelastic equations. SOWFA includes Schumann's boundary condition for surface stress (Schumann 1975) and boundary conditions for surface temperature flux or cooling rate (Basu et al. 2008). SOWFA can be run over flat or complex terrain. Because of the unstructured nature of the mesh, regions of increased refinement can be arbitrarily added where necessary, such as in turbine wakes and around regions of highly complex terrain. SOWFA models wind turbines using actuator lines, which are lines of body force rotating in space to simulate the impact of the turning wind turbine blades on the flow (Sørensen and Shen 2002).

In future years, the MMC team expects to transition to the Nalu microscale model, which is being designed to scale on exascale computers using next-generation computer architectures and will replace SOWFA in the DOE modeling suite. Development is taking place through the Exawind project (NREL 2018), a project within DOE's larger Exascale 

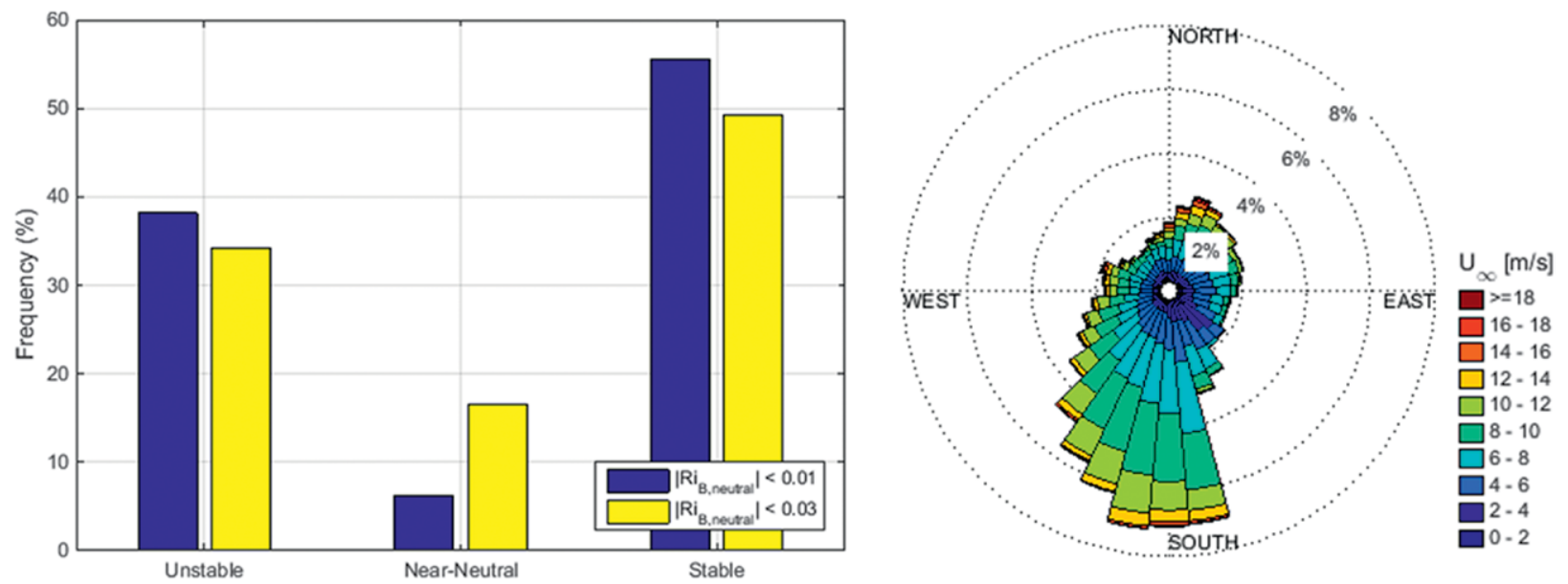

Fig. 2. Summary of characteristics of the SWiFT site for 730 days between 2012 and 2014. (a) Histogram of unstable, near-neutral, and stable conditions and (b) wind rose.

Computing Project (DOE 2018) and through A2e's High-Fidelity Modeling project. Nalu is being augmented to include many of the same meteorological and wind energy features as SOWFA.

SWiFT site data. The flat terrain validation efforts of the MMC project center around the SWiFT facility in West Texas. This site was chosen for its flat terrain with uniform land cover, relevance to wind energy applications, and the adjoining atmospheric facilities hosted by Texas Tech University's (TTU) National Wind Institute (NWI; Hirth and Schroeder 2014). Conditions are representative of the wind-energy-producing regions in the middle of the United States. The subset of NWI facilities used for this initial effort includes the 200-m meteorological tower, radar wind profiler, and data from the TTUoperated West Texas Mesonet. Raw data from the $200-\mathrm{m}$ tower are saved at a sample rate of $50 \mathrm{~Hz}$, with data dating back to July 2012. The tower has 10 sets of measurement booms ranging from 0.9 to $200 \mathrm{~m}$ in height, with sonic anemometers used to measure the mean wind speed and direction as well as turbulence parameters. Data from a radar wind profiler, located approximately $540 \mathrm{~m}$ to the southeast of the $200-\mathrm{m}$ tower, were used to determine the upper-level forcing that drives the microscale flow conditions as well as the upper-air conditions for the mesoscale model simulations. The atmospheric conditions at the SWiFT site were characterized using 730 days of the TTU historical dataset collected between 2012 and 2014, shown in Fig. 2, which is a histogram of the cases that are stable, neutral, and convective based on the computed bulk Richardson number calculated using gradients between the 2.4- and 10.1-m measurement stations. For the most stringent Richardson number criterion of neutral being within \pm 0.01 (the blue bars), note that neutral conditions occur only about $7 \%$ of the time, with unstable and stable conditions at $38 \%$ and $55 \%$, respectively. The wind rose of Fig. $2 b$ indicates a predominate southerly flow at the site.

WFIP 2 site data. Many wind plants are located in complex terrain; thus, it is important to consider modeling for such sites. The MMC project leverages data from the DOE WFIP 2 project documented in companion papers (Shaw et al. 2019; Wilczak et al. 2019; Olson et al. 2019; E. Grimit et al. 2019, unpublished manuscript). The WFIP 2 site in the Pacific Northwest of the United States observes a rich variety of complex atmospheric phenomena forced by the mountains, river valleys, and proximity to the Pacific coast, which was also the area of focus of a number of earlier studies (Yang et al. 2013; Yang et al. 2017; Berg et al. 2019). Gap flows, mountain waves, cold pools, meandering mountain wakes, thermal troughs and marine pushes, and additional phenomena were observed during the intensive operating period of the experiment conducted during 2016-17. The MMC team leveraged the detailed reporting and observations of the WFIP 2 team to identify cases to study that include these rich complex phenomena and to configure simulations to test the modeling and coupling strategies discussed below.

ADDRESSING THE CHALLENGES. As introduced in the first section and displayed in Fig. 1, several key challenges must be addressed for successful coupling across scales. This section discusses each and shows some results for specific case studies selected from the SWiFT and WFIP 2 datasets. 
Terra incognita. The boundary layer parameterizations of current mesoscale models assume that the grid does not resolve the turbulent eddies. When the grid spacing is decreased beyond some critical value, some aspects of the eddies may be within the grid resolution. Wyngaard (2004) called this the "terra incognita," which he estimated to be roughly between 100 - and 1,000-m grid spacing, where erroneous solutions, such as spurious rolls and erroneous distributions of turbulent kinetic energy (TKE) may develop.

The MMC team began investigating the impact of the terra incognita on coupled modeling of case studies at the SWiFT site by modeling flow at differing resolutions. The team documented evidence of impacts related to terra incognita issues using traditional subgrid turbulence closure approaches in the mesoscale simulations of $1-\mathrm{km}$ resolution and finer. Spurious horizontal rolls manifest in the wind speed (vertical and horizontal) as well as noise in the TKE, momentum flux, and temperature indicated these issues were present (Haupt et al. 2015).

A more detailed analysis documented that the upper limit of the terra incognita range should be based on the depth of the boundary layer (Rai et al. 2019). Figure 3 highlights the importance of including a mesoscale nest, which leads to more realistic simulations in the flow simulated with the microscale model. This figure shows three carefully constructed experiments with the outer mesoscale grid spacing for each equal to the depth of the boundary layer at that time $\left(z_{i}=1.6,2.3\right.$, and $3.2 \mathrm{~km}$ from bottom to top). No spurious rolls are evident as long as the grid spacing $(\Delta x y)$ is at the depth of the boundary layer, but spurious rolls become evident at grid spacing less than that depth. The results also suggest that mesoscale domains with grid spacing in the terra incognita do not have a large impact on the microscale LES results. That study leads to a series of guidelines for configuring coupled simulations (Rai et al. 2019).

- Users should avoid mesoscale domains that employ grid spacing smaller than the depth of the boundary layer due to potential development of unrealistic features in the flow at those grid spacings. The depth of the ABL defines the outer limit of the terra incognita.

- Development of spurious features in mesoscale WRF will not necessarily lead to incorrect model results in WRF-LES nests at finer resolutions that resolve the eddies, provided that a sufficiently large fetch is allowed for proper small-scale turbulence spinup and equilibration. Nevertheless, idealized simulations from Mazzaro et al. (2017) showed that the fetch required for turbulence development was larger for coarser mesoscale grid spacing.

- The finescale turbulence properties found on the microscale domain are nearly independent of the boundary layer parameterization (MYNN and YSU were tested) used on the mesoscale domains (Rai et al. 2019).

- The energy in WRF-LES nests coupled to a WRF mesoscale nest is greater than when the WRFLES simulation is driven by a reanalysis product, particularly at the larger wavelengths. a)

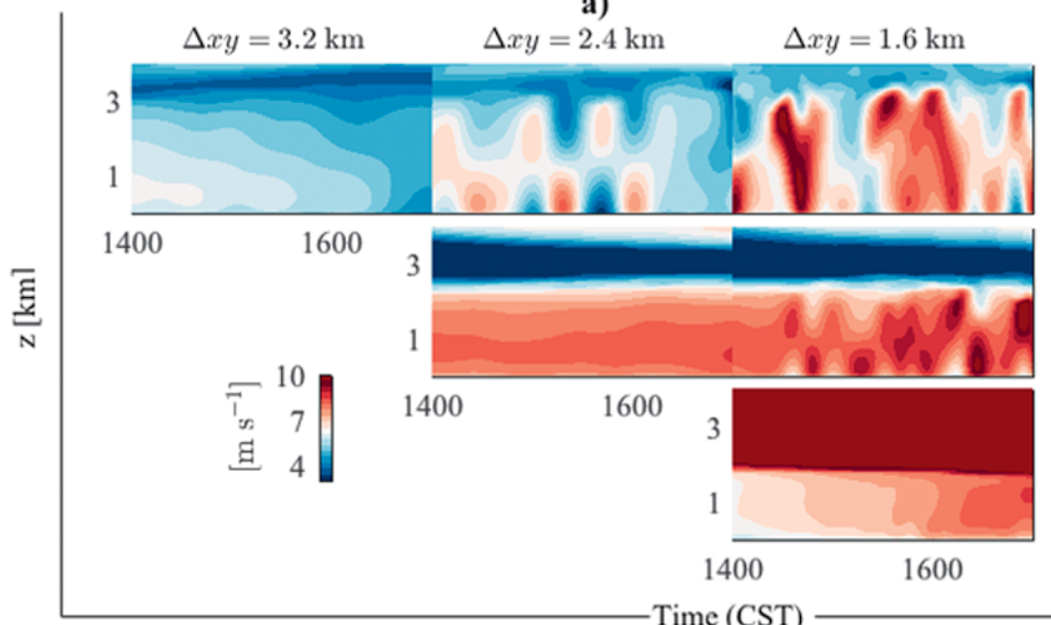

b)

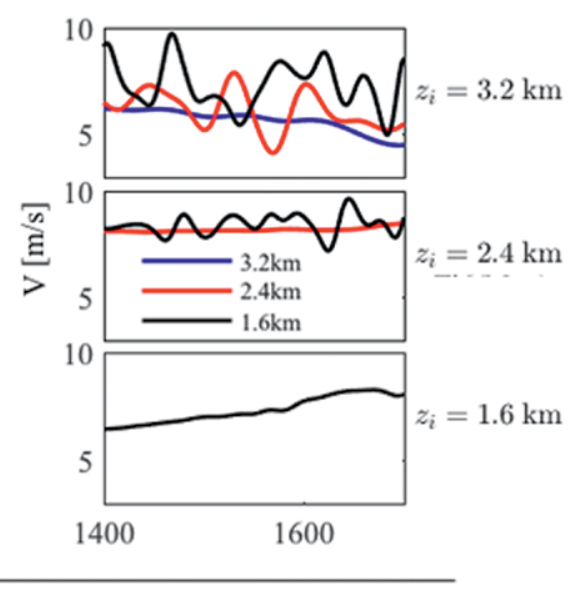

Fig. 3. Simulation results for (a) time-height contour constructed from the vertical profile of horizontal wind speed and (b) time series $(0.5 \mathrm{~km}$ above the surface) at the tower location of the SWiFT site obtained from simulations with three horizontal grid spacings and boundary layer depths (i.e., 3.2, 2.4, and $1.6 \mathrm{~km}$ ) (from Rai et al. 2019). 
The team also analyzed the impact of the terra incognita in complex terrain (Haupt et al. 2017), finding that the performance of the model depends on the site of interest and its situation within the complex terrain. The finer the grid spacing, the more realistic the terrain representation. For the case studied, simulated mountain waves were perpendicular to the flow and realistic, and no terra incognita issues were found, which was in contrast to results in flat terrain, in which unrealistic rolls formed parallel to the flow. This finding suggests that external forcing, such as from complex terrain, may outweigh the anomalous forcing from the mesoscale PBL scheme.

Coupling strategies. The MMC project seeks to discover best strategies for coupling the strengths of mesoscale models, which are designed to model the full nonstationary processes of the atmosphere, with those of the microscale LES models that can be used to optimize wind plant operations. Here we describe two primary methods being tested using WRF-LES and SOWFA as the microscale models.

The most straightforward way to incorporate mesoscale forcing into a microscale simulation is to use a unified computational model that supports both mesh refinement and scale-appropriate physics modules, such as WRF applied with its capability to include LES nests (WRF-LES). This method provides both the lateral boundary conditions and any internal forcing terms, including geostrophic wind forcing and its variability, as well as surface information, at the microscale domain model time step. Other physical forcing factors, such as large-scale advection, are automatically incorporated into the microscale domain.

The nesting approach used for the MMC WRF boundary-coupled simulations shown in Fig. 4 is similar to that presented by Rai et al. (2017), with six telescoping WRF Model domains (three mesoscale and three LES), beginning with the horizontal resolution of $12.15 \mathrm{~km}$ down to a resolution of $30 \mathrm{~m}$. The WRF mesoscale domains are run using a standard onedimensional boundary layer parameterization and horizontal grid spacings of $12.15,4.05$, and $1.35 \mathrm{~km}$. The LES domains (grid resolutions of 270, 90, and $30 \mathrm{~m}$ ) employ a standard three-dimensional subgridscale turbulence parameterization to represent features of the flow that remain subgrid at the mesoscale. Care has been used when making the jump from the mesoscale to LES domains to avoid having a grid with a resolution in the terra incognita, according to the guidelines proposed in the previous section. In this setup, the lateral boundary conditions for each nested domain are provided by the bounding domain.
Alternately, one may choose to use a more modular approach in which mesoscale information is extracted from the mesoscale model and then used to drive subsequent simulations in a separate microscale model. This modular approach is particularly useful for wind plant simulations where we wish to incorporate the mesoscale information with detailed representations of the turbines and their wakes that require a separate microscale model. This approach is necessary when coupling the mesoscale WRF and SOWFA, which is currently applied offline. We also choose to test this coupling method within WRF-LES to allow for direct comparison with the nesting method. Within this modular framework, there are multiple options.

One modular option begins with an LES with periodic boundary conditions as a precursor to generate initial and boundary conditions for a subsequent nonperiodic wind plant LES. The periodic precursor simulation spins up turbulence very quickly and can include surface heat flux or skin temperature information along with height- and time-varying boundary and internal source-term forcings derived from the mesoscale WRF simulation or from observations. This option allows the precursor to include mesoscale influence, covering a wider variety of possible conditions. Generally, the source-term forcings extracted from mesoscale WRF are extracted from one grid column or are the average over a few grid columns surrounding the region of interest.

The second modular option provides internal source-term forcing derived from the time rate of change, or tendency, of the equations of motion of the mesoscale model. In this internal forcing option, time series of flow data are extracted from the WRF flow field on surfaces corresponding to the SOWFA domain boundaries. This includes velocity and potential temperature on the lateral and top boundaries and surface sensible heat flux or skin temperature on the surface boundary. These boundary data, along with the pressure gradient force over the extent of this microscale domain, are used to drive the microscale simulation. The mesoscale tendency $U_{\text {tend }}$ is broken into its different contributions, which include advection $U_{\text {adv }}$, Coriolis acceleration $U_{\text {cor }}$, the pressure gradient force $U_{\mathrm{pg}}$, subgrid-scale effects $U_{\text {sgg' }}$, and external forces $U_{F}$ :

$$
U_{\text {tend }}=U_{\text {adv }}+U_{\text {cor }}+U_{\mathrm{pg}}+U_{\mathrm{sgs}}+U_{F}
$$

These individual contributions to the tendency can be extracted from the mesoscale WRF simulation and subsequently used to drive the microscale simulation. For momentum, the pressure-gradient force and 
mesoscale advection term are applied as sources to the microscale momentum equation. For potential temperature, the combination of the mesoscale advective term and surface heat flux or skin temperature is applied to the microscale potential temperature transport equation. With this method, the microscale domain is periodic, meaning that without coupling, the only way for temperature to be transported into the domain is through surface flux. However, when we include a mesoscale advection source term on the interior of the microscale flow field, the effect of relatively warmer or cooler air masses advecting into the domain can be replicated in the periodic domain. This method is derived from those demonstrated by Zajaczkowski et al. (2011) and Rodrigo et al. (2016). This internal forcing option supplies the microscale model with short-duration mean (e.g., 10-min means) vertical profiles of velocity and potential temperature. The microscale model can then be driven by the horizontally planar-averaged mean LES solution toward the given profiles.

The idea of using mesoscale-derived, internal source terms was tested within a periodic offline simulation using WRF-LES, where the geostrophic wind components and advective horizontal velocity and temperature sources were extracted from the same mesoscale simulations used to force nested simulations. The mesoscale forcing parameters were allowed to vary in the vertical direction; however, homogeneity was enforced within each horizontal plane to obey the constraints imposed by the use of periodic boundary conditions. Strictly, when the horizontal pressure gradient varies in the vertical direction, the assumption of horizontal homogeneity is violated; however, the resulting error over a relatively small domain may be negligible (Brown 1996, 1999; Sorbjan 2004; Fedorovich et al. 2017).

We show some results for a nonstationary case study from the SWiFT site: 8 November 2013. This case represents a classic diurnal cycle where the stable boundary layer breaks up in the morning and the scale of the turbulence grows as the surface is heated, developing into a fully convective boundary layer by midafternoon. As the sun sets and heating stops, the convective layer breaks down and transitions through a neutral layer to a stable nocturnal layer, complete with a nocturnal low-level jet (LLJ).
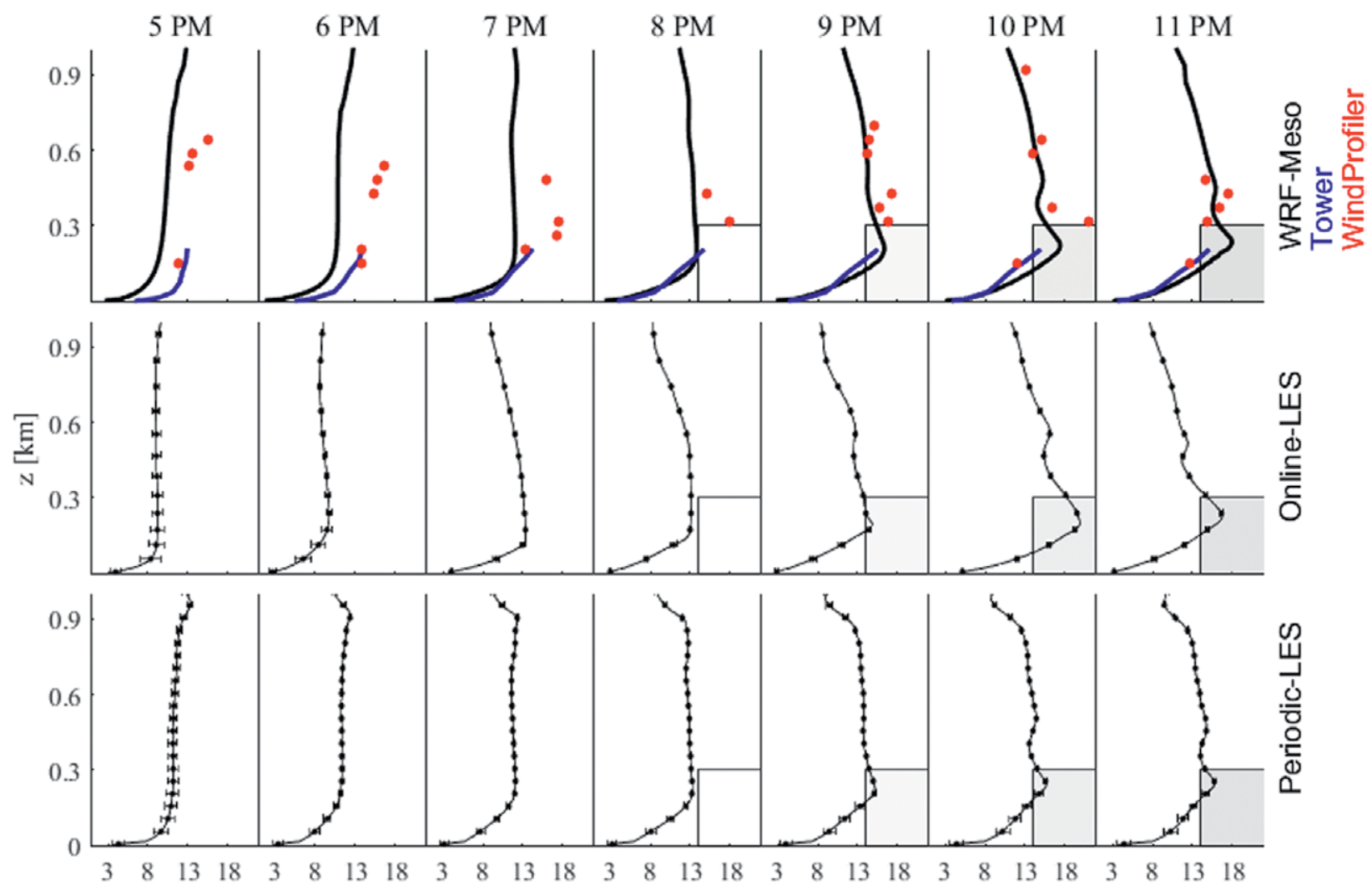

Fig. 4. Vertical profiles of wind speed during (left to right) 1700-2300 LT $8 \mathrm{Nov}$, as an LLJ develops from the (top) WRF mesoscale, (middle) online coupled LES, and (bottom) offline periodic LES with the noise removed from the lower portion of the geostrophic wind profiles. The boxes indicate where the turbines are expected to perform at full capacity. 
Figure 4 compares vertical profiles of wind speed each hour during the evening transition and development of the LLJ on 8 November from three simulations: the WRF mesoscale (top), the online coupled LES (middle), and the offline periodic LES (bottom). The offline periodic LES is meant to mimic the behavior of coupling the mesoscale WRF (constructed using $5 \times 5$ grid cells) to any other stand-alone LES model. For that run, the constant values of the geostrophic wind speeds, computed from just above the ABL height during the WRF mesoscale simulations, are applied throughout the depth of the ABL in order to remove spurious noise, but also removed some of the real atmospheric forcing required to capture the correct evolution of the LLJ. The boxes in Fig. 4 indicate wind speeds greater than $14 \mathrm{~m} \mathrm{~s}^{-1}$ between the surface and $300 \mathrm{~m}$, indicating that the turbines are expected to produce power at their full rated capacity. The horizontal bars superimposed on the LES wind speed profiles indicate two standard deviations of the resolved variability within the appropriate (finest) domain over each hour of planar averaging. The offline periodic LES is seen to produce an LLJ that is weaker than either the online coupled or the mesoscale simulations, with a difference of approximately $5 \mathrm{~m} \mathrm{~s}^{-1}$ at the jet nose at 2000 CST. The weaker LLJ in the offline coupled LES underscores the importance of online inclusion of the details of baroclinicity and advection within the ABL. Thus, we conclude that for this case, the online simulation produced a much better development of the LLJ than the offline simulation.

This modular mesoscale-derived, internal-sourceterm approach was also tested in SOWFA. The mesoscale-derived source terms are allowed to vary in height and in time, but are held constant in both horizontal directions, due to the use of periodic boundary conditions. Examples of the $80-\mathrm{m}$ planar views of horizontal velocity appear in Fig. 5 . We see the finescale turbulence at the beginning of the day in Fig. 5a. By 1000 LST, the flow has evolved into longitudinal rolls (Fig. 5b). A combination of wind speed and surface heat flux as well as boundary layer height results in the ratio of mixed-layer height and Monin-Obukhov length $\left(z_{i} / L\right)$ in the range between 0 and -25 . For these values of $z_{i} / L$ over flat terrain, convective rolls are expected (LeMone 1976; Weckwerth et al. 1999). In these LES results, such convective rolls are well resolved. As heating progresses, hexagonal cells form, as seen in Fig. 5c. As heating dies down toward 1700 LST, the flow transitions back toward rolls (Fig. $5 \mathrm{~d}$ ), then to finer-scale turbulence (Fig. 5e). This case demonstrates the ability of this technique to capture the changes in turbulence characteristics forced by the mesoscale flow and by surface heating, with the microscale model following the dynamic changes imposed at the mesoscale.

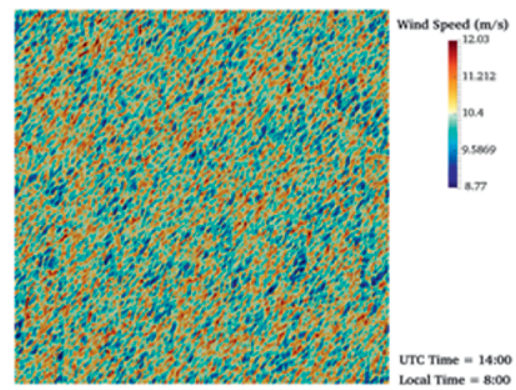

a

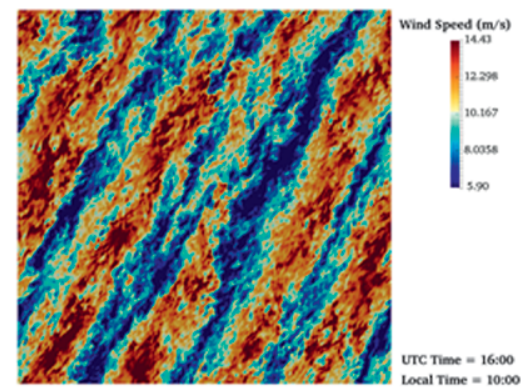

b

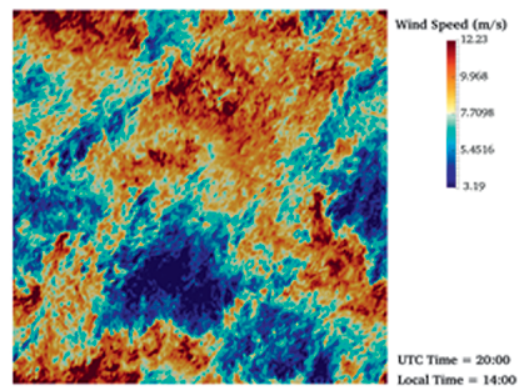

C

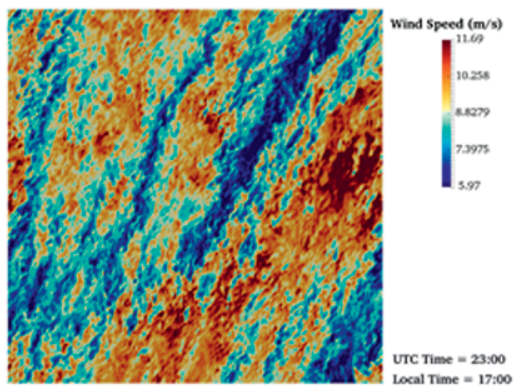

d

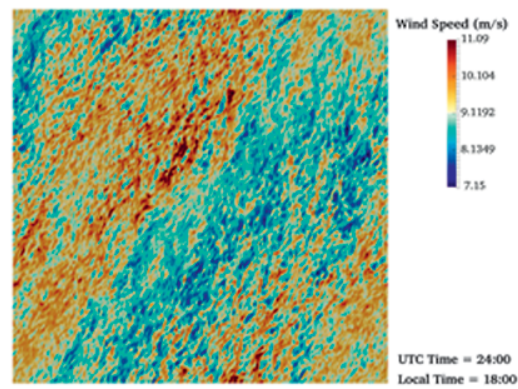

e

FIG. 5. Horizontal slices at $80 \mathrm{~m}$ above the surface of instantaneous horizontal velocity from the SOWFA SWiFT at (a) 0800, (b) 1000, (c) 1400, (d) 1700, and (e) 1800 LT 8 Nov simulation. The different turbulence characteristics at different times of the day have evolved in response to the mesoscale forcing. 
Generating turbulence. Mesoscale models are not run at grid resolutions that allow resolving boundary layer turbulence. In contrast, the microscale models are constructed to specifically resolve that turbulence. Thus, when a microscale simulation is forced by data from a mesoscale simulation, one must spur the turbulence at the correct scales. Typically, turbulence forms slowly within the LES domain, requiring long fetches for the turbulence to develop that imposes a computational overhead. The MMC project team has examined several approaches to accelerating turbulence development, including 1) methods that perturb the inflow, either stochastically or with spectral turbulence information, and 2) precursor methods, for which turbulence information is computed from a separate offline simulation.

The first approach superimposes small-amplitude perturbations onto the resolved inflow variables (Mirocha et al. 2014; Muñoz-Esparza et al. 2014). The approach is motivated by the idea that perturbations of the flow field at optimal spatiotemporal scales and magnitudes will trigger turbulence development through the nonlinearities inherent in the governing flow equations. Given that these perturbations and resulting correlations develop within a given flow environment, defined, for example, by surface roughness and flux, ABL structure, and wind speed and direction profiles, the perturbations may more quickly lead to a turbulence state that is consistent with that environment. The stochastic cell-perturbation method (SCPM; Muñoz-Esparza et al. 2014, 2015; Muñoz-Esparza and Kosović 2018) has focused on perturbations in the potential temperature field. The team has also investigated application of perturbations to the velocity field as a variant of the SCPM, imposed through tendencies in the momentum equations. The use of momentum tendencies showed somewhat similar performance to the original potential temperature perturbations in the SCPM, with the temperature-based SCPM reaching equilibrium for the entire spectrum of turbulent eddies more efficiently. Potential temperature perturbations were found to be more effective in convective conditions (Mazzaro et al. 2019). Despite some differences, these findings point to the robustness of the cell perturbation method.

The SCPM was tested in case studies involving full physics multiscale coupled simulations within the WRF Model. The SCPM was implemented to run concurrently with the nested LES domains, using relevant mesoscale information passed to the nested LES domain(s) from the finest mesoscale domain within one coupled simulation. This SCPM method was examined using the 8 November 2014 diurnal cycle at the SWiFT facility, simulated both with and without the SCPM, as displayed in Fig. 6. Simulations revealed that the SCPM improved the simulations for all time periods. For the more convective conditions at 1200 CST, the improvement was primarily in reducing the fetch required for small-scale turbulence development (note that underresolved convective structures were present at the inflow at that particular time). Moreover, the SCPM considerably improved the representation of turbulence during the neutral and stable conditions later in the afternoon and overnight, as seen in the figure and in plots of quantities including wind speed, TKE, and friction velocity (not shown; see Haupt et al. 2017). It is worth remarking that as Muñoz-Esparza and Kosović (2018) demonstrated, the existence of a convective $\mathrm{ABL}$ does not always guarantee rapid onset of turbulence in the nested LES domain. The authors showed that, in fact, the transition fetch is governed by the ratio of convective velocity scale to the mean horizontal advection, and can often lead to significant development regions during daytime conditions.

As an alternative to the SCPM, the MMC team investigated two different synthetic turbulence perturbations approaches to enrich the inflow turbulence spectra, and consequently accelerate the equilibration of turbulence statistics: TurbSim and Gabor Kinematic Simulation. TurbSim, an established stochastic turbulence simulator used in wind turbine aeroelastic modeling, explicitly specifies a spectral model and includes additional parameters for specifying turbulence intensity and spatial coherence (Kelley 2011). The Gabor method uses discrete Gabor modes to simulate small-scale turbulence and models nonstationary mesoscale forcings, Coriolis effects, stratification, and heterogeneity (Ghate and Lele 2017). The team tested both enrichment methods under idealized neutral conditions, comparing to a baseline high-resolution microscale ABL simulation that captures the transition to fully developed turbulence without added perturbations. We found that when applied to coarse-resolution microscale ABL simulations-with grid spacing increased by up to a factor of 4 -both methods can recover the same turbulence spectra as the baseline. The fetch in the enriched cases was reduced by a factor of 4 compared to an unenriched simulation (Quon et al. 2018). The team continues to extend these methods to more complex cases.

The second approach to generating turbulence involved precursor simulations. An example experiment used a periodic LES driven by mesoscale forcing parameters to provide turbulent inflow to an offline microscale simulation over complex terrain. The method requires precomputation of both 

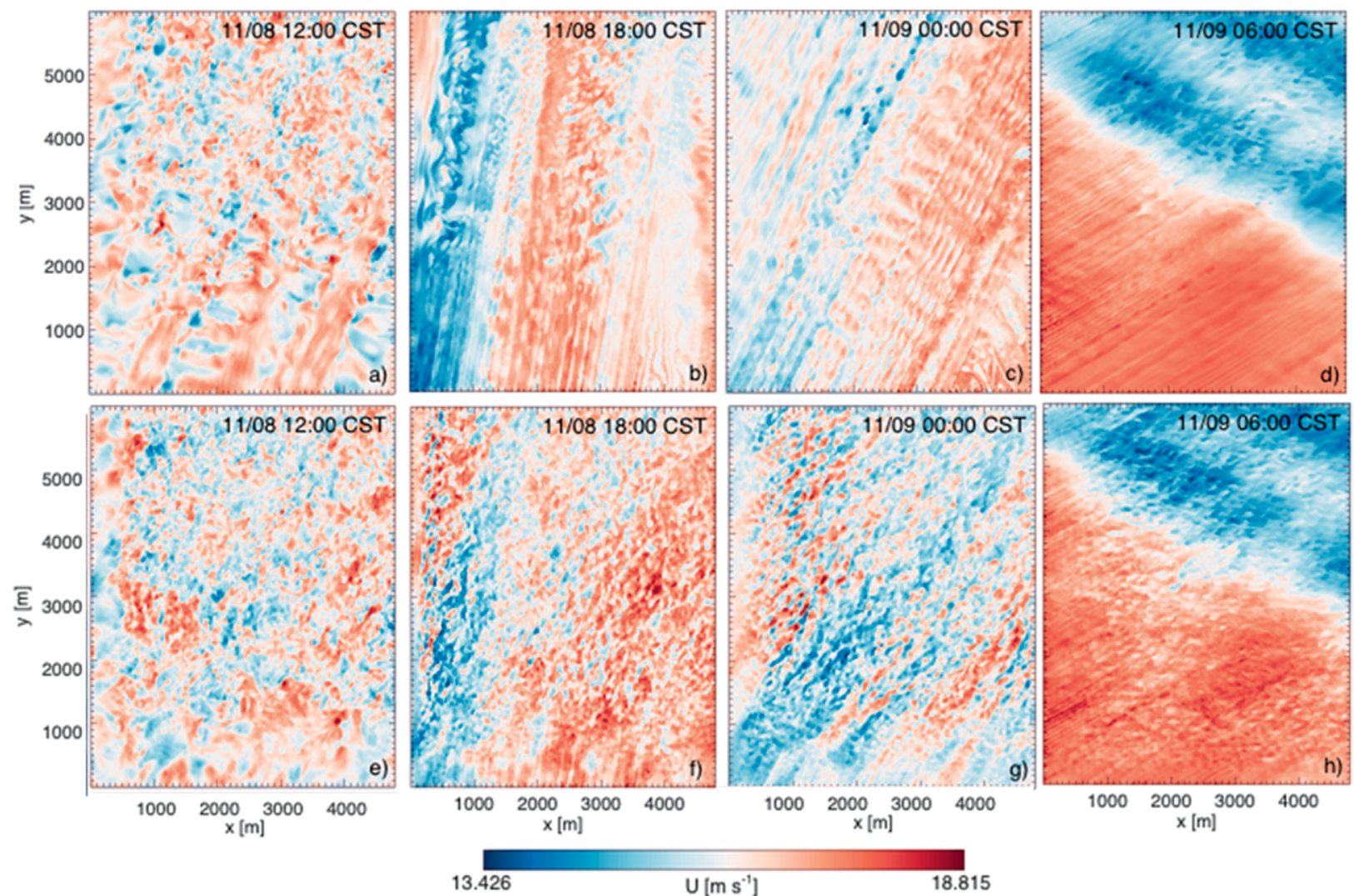

$\mathrm{U}\left[\mathrm{m} \mathrm{s}^{-1}\right]$

18.815

FIG. 6. Planar view at $100 \mathrm{~m}$ of LES with stochastic cell perturbations applied in a case study of a diurnal cycle for 8 and 9 Nov 2013 at the SWiFT site. Columns display the zonal velocity field at a particular time of day: (a),(e) I 200 LT 8 Nov, (b),(f) 1800 LT 8 Nov, (c),(g) 0000 LT 9 Nov, and (d),(h) 0600 LT 9 Nov. Rows show simulations (a)-(d) without SCPM and (e)-(h) with SCPM.

the mesoscale forcing and the microscale turbulence field, which is computationally expensive, as well as high-frequency storage of model output from the precursor run. The subsequent wind plant simulation often uses turbine-local grid refinement, so the precursor may be significantly less computationally expensive than the wind plant simulation. However, once those simulations are complete, the offline microscale simulation does not require a turbulence generation fetch upwind of an area of interest. This approach was successfully applied over complex terrain at the WFIP 2 Physics Site, showing great promise as a means of providing instantaneous turbulent inflow (Haupt et al. 2019).

Boundary and initial conditions. Because WRF includes a land surface model while stand-alone LES models like SOWFA do not, those stand-alone LES models cannot estimate time-varying fluxes consistent with the flow. We wish to consider how this will impact the flow at the microscale and its turbulence characteristics. To assess this issue, we tested a set of simulations using WRF-LES nested inside a mesoscale
WRF domain for a case at the SWiFT site. The first simulation applies the interactive WRF land surface model to provide temporally and spatially varying surface fluxes. The second simulation uses surface fluxes that are averaged over the domain for that time step. This approach assures that the energy input is the same in both simulations. Differences in the instantaneous wind speed and vertical velocity at $80 \mathrm{~m}$ above ground level in the two simulations are shown in Fig. 7. Clearly, the differences in the surface fluxes are expected (top-left row of Fig. 7). There are also differences in both the vertical velocity (as large as $\pm 4 \mathrm{~m} \mathrm{~s}^{-1}$ ) and horizontal wind speed (also as large as $\pm 4 \mathrm{~m} \mathrm{~s}^{-1}$ ). The middle-left and bottom-left rows of Fig. 7, however, indicate very little change in the vertical and horizontal velocities. In addition, the spectra in the right column confirm similarity between the two cases. This result suggests that providing timevarying yet spatially homogeneous surface conditions is a promising technique to use for coupling the mesoscale to the microscale.

The MMC team has emphasized simulating cases from field campaigns, such as from the WFIP 


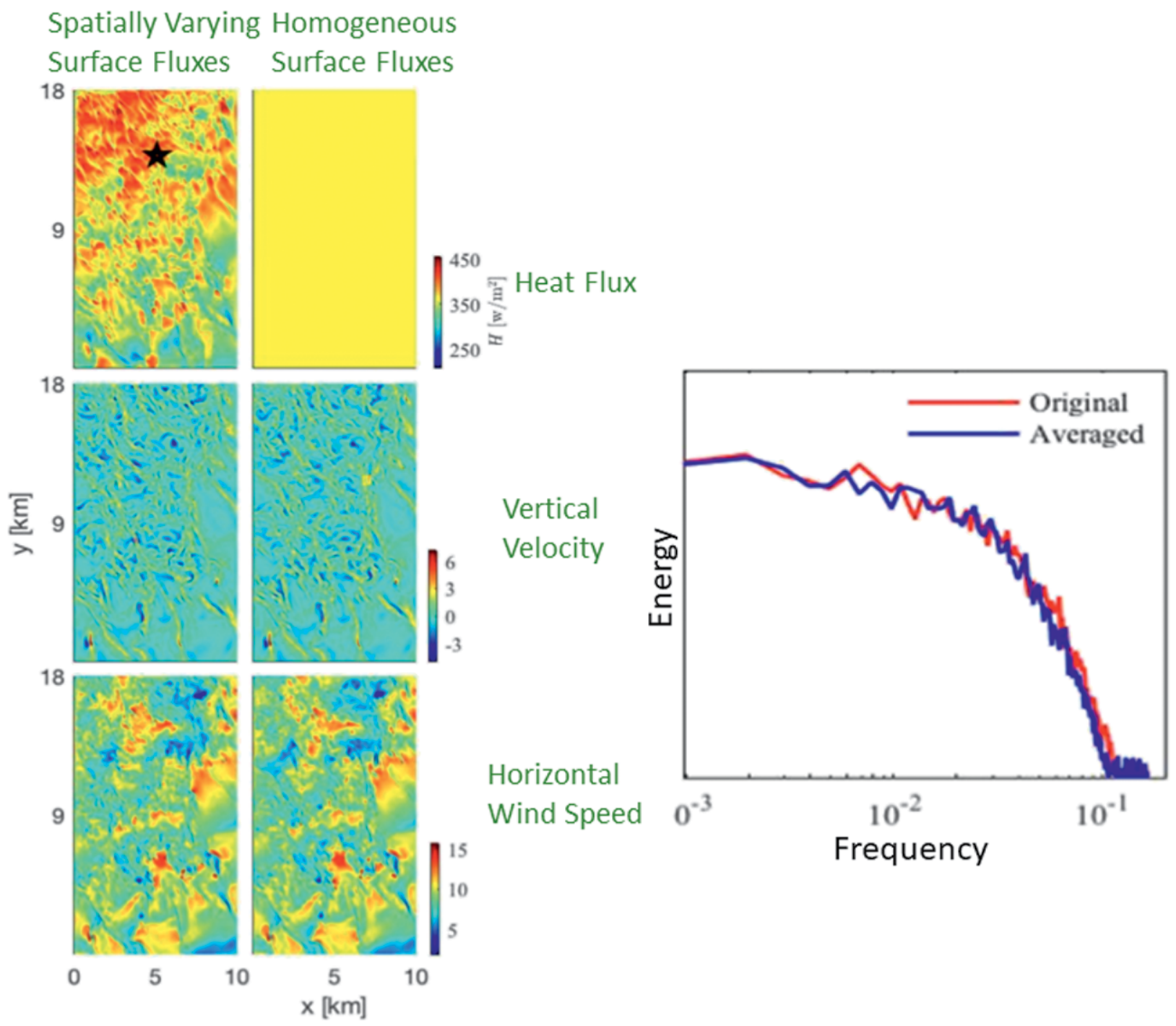

FIG. 7. (left) Instantaneous (top) heat fluxes, (middle) vertical velocity, and (bottom) wind speed with spatially varying fluxes, homogeneous surface fluxes, and differences between the two sets of simulations. (right) Spectra comparing the velocity spectrum from the original surface conditions (red) with the spatially averaged one (blue).

2 observational experiment in complex terrain. An example case using observations at the WFIP 2 Physics Site from 21 November 2016 was characterized by topographic wake and mountain waves over the area. The mesoscale-to-microscale simulation was carried out using WRF's nesting capability; the parent nest was run in mesoscale mode while two inner nests were run in LES mode. The outer mesoscale domain is $\sim 500 \mathrm{~km}$ $\times 500 \mathrm{~km}$, whereas the two nested LES domains are $123 \mathrm{~km} \times 92 \mathrm{~km}$ and $6.8 \mathrm{~km} \times 6.8 \mathrm{~km}$, respectively. The Obs. (Physics) Site is situated $5 \mathrm{~km}$ downstream from the west face of the innermost domain, allowing sufficient fetch for the westerly flow. Initial and boundary conditions for the simulation were provided by the High-Resolution Rapid Refresh forecasting system (Benjamin et al. 2016), based on the WRF mesoscale model, which was enhanced under the WFIP 2 project for these conditions (Olson et al. 2019). The HRRR forecast over the Columbia River gorge area for the period of interest indicated overall skillful prediction (not shown), indicating that it would be a good case for comparison, although the increase in wind speed at around 1900 UTC (1100 LT) lagged by about an hour in the simulation. Thus, large wind speed errors at the beginning of the simulation resulted in large differences between simulated and observed TKE and turbulent stress at hub height, but the difference decreased over the $2 \mathrm{~h}$ of the simulation. Figure 8 displays the LES simulation at $143-$ and $13-\mathrm{m}$ resolutions. The mountain waves and meandering wake in the lee of Mt. Hood are striking. Spectral analysis showed promising agreement at frequencies corresponding to boundary layer-scale eddies (Fig. 8c). Good agreement indicates that even when the mesoscale flow is not captured 


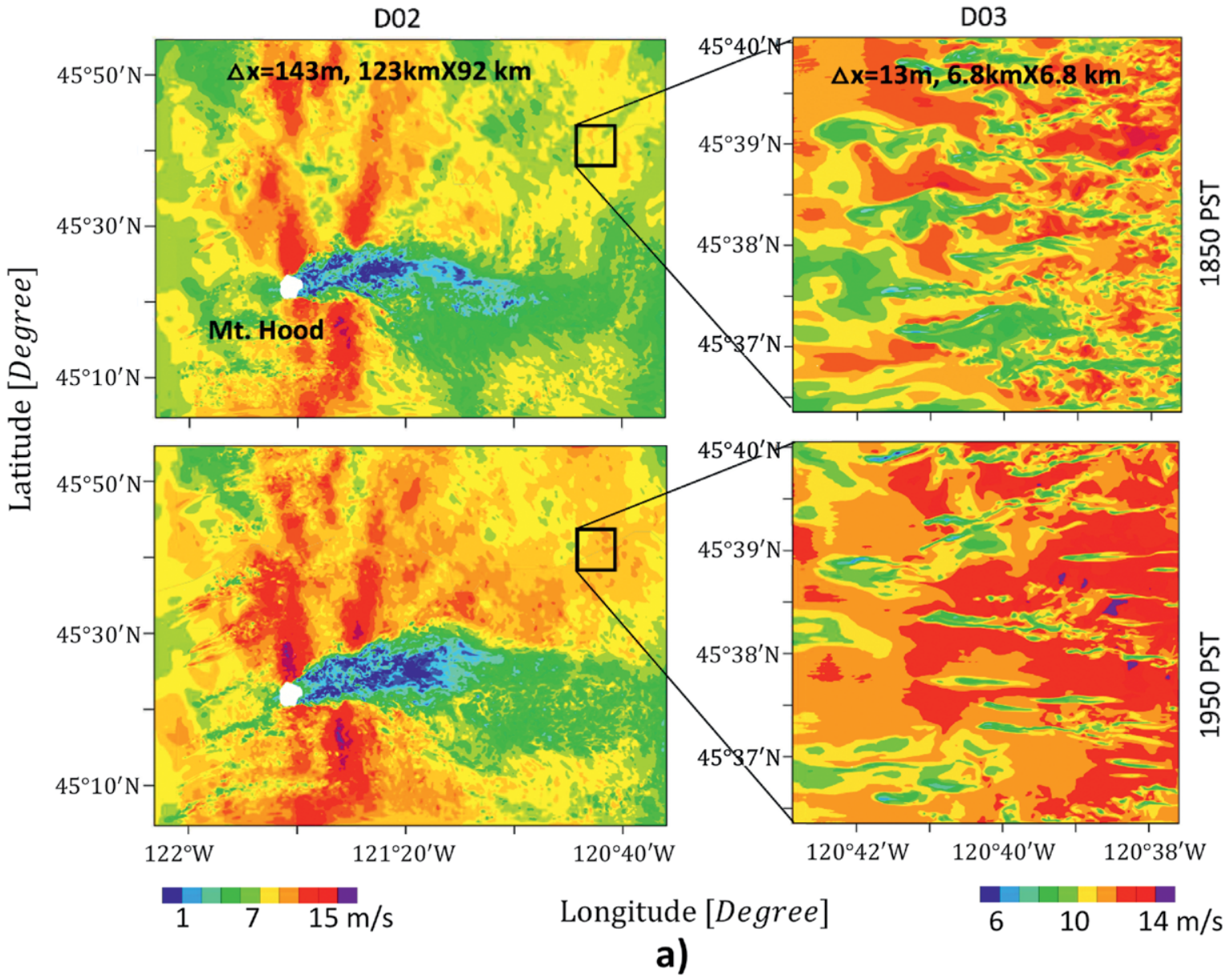

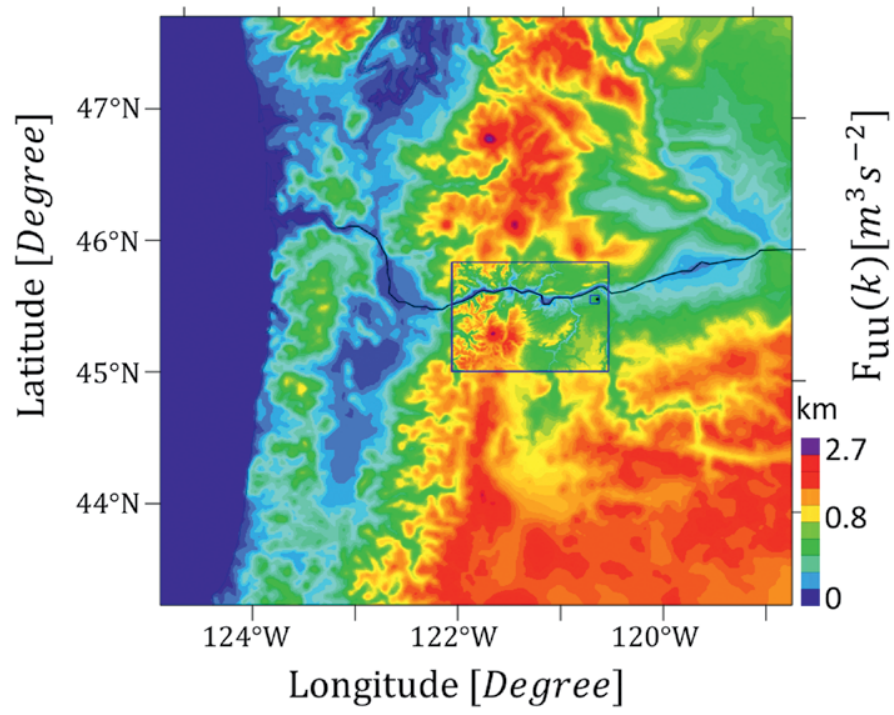

b)

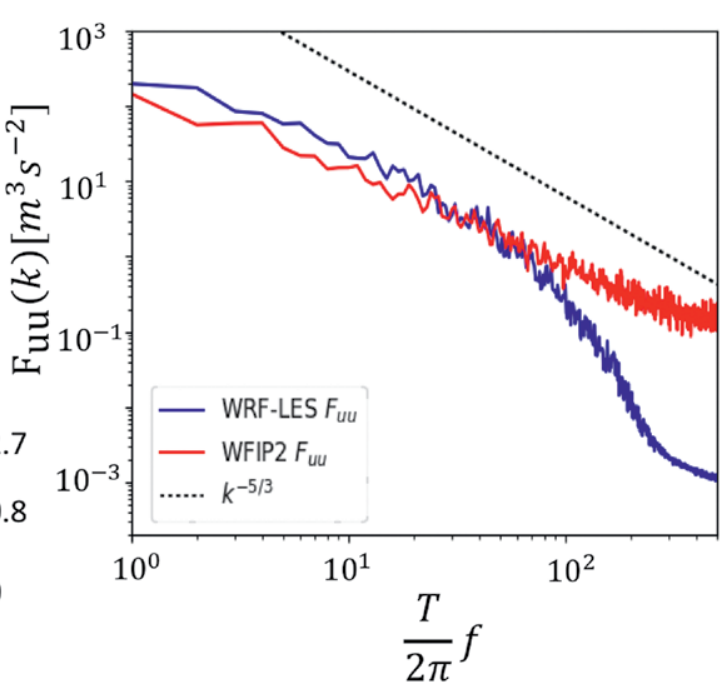

c)

FIG. 8. Results of modeling complex flow in the Columbia River valley: (a) two horizontal slices I h apart at (left) 2,100 $\mathrm{m}$ above ground level for domain 2 (143-m grid spacing) and (right) $100 \mathrm{~m}$ above ground level for domain 3 (I3-m grid spacing), (b) model domain, and (c) energy spectra comparing WRF-LES with measured spectra.

accurately, the turbulent energy transfer from large turbulent production scales to smaller scales can be represented accurately in a well-resolved LES.
Assessment and uncertainty quantification. This paper has demonstrated the team's philosophy of grounding the assessment in data derived from case studies 
selected from field observational programs in different climatological conditions and during nonstationary events. The metrics selected include those most relevant to wind energy, including wind speed and direction profiles, shear and veer across the rotor diameter, velocity spectra at different heights, and profiles of turbulent kinetic energy. The team is moving toward using power from wind turbines as an additional metric in future work (Haupt et al. 2019).

Industry colleagues have expressed interest in quantifying the uncertainty in the modeling results. It is important to consider multiple types of uncertainty in doing so. The inherent uncertainty of the nonlinear, dissipative chaotic flow is structural in nature. That is in contrast to uncertainty in the modeling choices, both in terms of parameters used in setting up the models as well as the boundary conditions. Note that uncertainty in the wind speed can be magnified when estimating uncertainty in the power produced, particularly for the steep region of the power curve where the relationship is cubic. More information on uncertainty quantification techniques is found in the team's annual reports (Haupt et al. 2017, 2019) and in Yang et al. (2017). Note, however, that a major uncertainty in the microscale simulation is whether the mesoscale captures the larger-scale processes correctly. In particular, it can be challenging to correctly capture the timing of dynamic events.

CONCLUSIONS. Coupling the mesoscale to the microscale flow enables simulations to capture the richness of atmospheric flow that drives the energetics to be derived from the wind to generate electricity. This team of national laboratories has made substantial progress in defining the best ways to integrate the advantages of both mesoscale and microscale models. A sampling of some of the results has been discussed herein. Some accomplishments of this project include the following:

- Established metrics for validation of these models relevant to wind plant simulations and the coupling mechanism, including evaluating turbulence.

- Determined that users should avoid driving microscale simulations with large-scale reanalysis products alone. Fully coupled simulations that employ mesoscale nests contain more energy at larger wavelengths than microscale simulations driven by a reanalysis product alone.

- Developed, tested, and evaluated methods to deal with spurious rolls resulting from models with grid spacing in the terra incognita. Showed that the upper end of the terra incognita is roughly equal to the boundary layer depth. Found that in most cases it is possible to configure WRF to skip grid spacings in the terra incognita (Rai et al. 2019; Haupt et al. 2017).

- Developed, tested, and evaluated two general approaches (with several methods for each in two sets of models) to couple mesoscale-to-microscale simulations, each with different advantages and disadvantages and noted that applicability may be situationally dependent.

- Developed, tested, and evaluated two flavors of the SCPM method to initialize turbulence in the microscale models that is subgrid to the mesoscale models, finding that this type of stochastic perturbations can efficiently induce forcing-consistent turbulence at the correct scales.

- Demonstrated and evaluated coupled simulations over complex terrain leveraging the rich WFIP 2 dataset, capturing the complexity of the flow in mountainous terrain (Fig. 7)

- Explored methods to better represent the surface layer in both mesoscale and microscale simulations using canopy models of varied complexity (not shown here; see Haupt et al. 2019).

These results have been presented to the community through a series of articles in the peer-reviewed literature (Rai et al. 2017, 2019; Mirocha et al. 2018; Rodrigo et al. 2016; Muñoz-Esparza et al. 2017; Quon et al. 2018); through presentations at conferences including those of the American Meteorological Society, WindTech, Torque, and International Conference on Energy and Meteorology; and a series of industry teleconferences and workshops. During the first phase of this project, the work emphasized atmospheric flow without including wind turbines. Much work remains, however, to develop and optimize a robust, fully coupled modeling system that can be used across industry. To provide publicly available data, the results of MMC modeling and case studies are being archived in DOE's Data Archive and Portal (DAP; https://a2e.energy.gov/data). The team welcomes collaborations with researchers doing similar work and seeks to share results and methodologies.

As computations move toward exascale machines, it will become more feasible to perform higher-resolution simulations and LES may become feasible in near-real time. To that end, the team is moving toward developing, validating, and distributing software tools that will enable further technological developments in wind energy. A version of WRF that includes the facilities required to better model the details of flow in wind plants is being developed. The Nalu microscale model is being optimized to include the ability to model finescale 
flow of wind plants. The best-practices methodology to couple and configure WRF and Nalu is being assessed and documented. These exascale-enabled fully coupled systems will be valuable for wind resource assessment, micrositing of wind turbines within the plant to optimize power production, and optimizing operations through better control of individual turbines. It will provide a simulation environment to enable wind plant technology innovation, and enrich forecasting to include these finescale features. The team seeks to advance the modeling technologies so that when these exascale machines become available, we are poised to best leverage their capabilities to improve modeling for better harvesting wind energy. Moreover, these methodologies are also valuable in validating and informing the lower-order models and quantifying the uncertainty in our modeling approaches.

ACKNOWLEDGMENTS. Funding for this work was provided by the U.S. DOE Office of Energy Efficiency and Renewable Energy Wind Energy Technologies Office. This work was authored (in part) by the National Renewable Energy Laboratory, operated by Alliance for Sustainable Energy, LLC, for the U.S. Department of Energy (DOE) under Contract DE-AC36-08GO28308. Funding provided by the U.S. Department of Energy Office of Energy Efficiency and Renewable Energy Wind Energy Technologies Office. The views expressed in the article do not necessarily represent the views of the DOE, NSF, or the U.S. Government. The U.S. Government retains and the publisher, by accepting the article for publication, acknowledges that the U.S. Government retains a nonexclusive, paid-up, irrevocable, worldwide license to publish or reproduce the published form of this work, or allow others to do so, for U.S. Government purposes. The National Center for Atmospheric Research is sponsored by the National Science Foundation. The authors thank three anonymous reviewers for thoughtful comments that helped us improve the manuscript.

\section{REFERENCES}

American Wind Energy Association, 2018: Wind energy facts at a glance. Accessed 1 November 2018, www .awea.org/wind-101/basics-of-wind-energy/wind -facts-at-a-glance.

Basu, S., A. A. M. Holtslag, B. J. H. Van De Wiel, A. F. Moene, and G.-J. Steeneveld, 2008: An inconvenient "truth" about using sensible heat flux as a surface boundary condition in models under stably stratified regimes. Acta Geophys., 56, 88-99, https://doi .org/10.2478/s11600-007-0038-y.

Benjamin, and Coauthors, 2016: A North American hourly assimilation and model forecast cycle: The
Rapid Refresh. Mon. Wea. Rev., 144, 1669-1694, https://doi.org/10.1175/MWR-D-15-0242.1.

Berg, L. K., Y. Liu, B. Yang, Y. Qian, J. Olson, M. Pekour, P.-L. Ma, and Z. Hou, 2019: Sensitivity of turbineheight wind speeds to parameters in the planetary boundary-layer parameterization of the Weather Research and Forecasting Model: Extension to wintertime conditions. Bound.-Layer Meteor., 170, 507-518, https://doi.org/10.1007/S10546-018-0406-y.

Brown, A. R., 1996: Large-eddy simulation and parameterization of baroclinic boundary layer. Quart. J. Roy. Meteor. Soc., 122, 1779-1798, https://doi.org/10.1002 /qj.49712253603.

_-, 1999: Large-eddy simulation and parameterization of the effects of shear on shallow cumulus convection. Bound.-Layer Meteor., 91, 65-80, https://doi .org/10.1023/A:1001836612775.

Cheng, W. Y. Y., Y. Liu, A. J. Bourgeois, Y. Wu, and S. E. Haupt, 2017: Short-term wind forecast of a data assimilation/weather forecasting system with wind turbine anemometer measurement assimilation. Renewable Energy, 107, 340-351, https://doi.org /10.1016/j.renene.2017.02.014.

Daniels, M. H., K. A. Lundquist, J. D. Mirocha, J. D. Wiersema, and F. K. Chow, 2016: A new vertical grid nesting capability in the Weather Research and Forecasting (WRF) Model. Mon. Wea. Rev., 144, 3725-3747, https://doi.org/10.1175/MWR-D-16 -0049.1 .

DOE, 2018: Exascale computing project. U.S. Department of Energy Office of Science, accessed November 2018, www.exascaleproject.org/.

Fedorovich, E., J. A. Gibbs, and A. Shapiro, 2017: Numerical study of nocturnal low-level jets over gently sloping terrain. J. Atmos. Sci., 74, 2813-2834, https:// doi.org/10.1175/JAS-D-17-0013.1.

Fernandez-Gonzalez, S., M. L. Martin, E. Garcia-Ortega, A. Merino, J. Lorenzana, J. L. Sanchez, F. Valero, and J. Sanz Rodrigo, 2018: Sensitivity analysis of the WRF Model: Wind-resource assessment for complex terrain. J. Appl. Meteor. Climatol., 57, 733-753, https://doi.org/10.1175/JAMC-D-17-0121.1.

Fitch, A. C., J. B. Olson, J. K. Lundquist, J. Dudhia, A. K. Gupta, J. Michalakes, and I. Barstad, 2012: Local and mesoscale impacts of wind farms as parameterized in a mesoscale NWP model. Mon. Wea. Rev., 140, 30173038, https://doi.org/10.1175/MWR-D-11-00352.1.

Fleming, P. A., and Coauthors, 2014: Evaluating techniques for redirecting turbine wakes using SOWFA. Renewable Energy, 70, 211-218, https://doi .org/10.1016/j.renene.2014.02.015.

_ - and Coauthors, 2015: Simulation comparison of wake mitigation control strategies for a two-turbine 
case. Wind Energy, 18, 2135-2143, https://doi.org/10 $.1002 /$ we.1810.

Ghate, A. S., and S. K. Lele, 2017: Subfilter-scale enrichment of planetary boundary layer large eddy simulation using discrete Fourier-Gabor modes. J. Fluid Mech., 819, 494-539, https://doi.org/10.1017/jfm.2017.187.

Haupt, S. E., and Coauthors, 2015: First year report of the A2e Mesoscale to Microscale Coupling Project. Pacific Northwest National Laboratory Rep. PNNL25108, 124 pp.

— , and Coauthors, 2017: Third year report of the Atmosphere to Electrons Mesoscale to Microscale Coupling Project. Pacific Northwest National Laboratory Rep. PNNL-28259, 137 pp.

- , and Coauthors, 2019: FY18 report of the Atmosphere to Electrons Mesoscale to Microscale Coupling Project. Pacific Northwest Laboratory Rep. PNNL-26267, 124 pp.

Hirth, B., and J. Schroeder, 2014: A summary of the National Wind Institute Meteorological Measurement facilities at the Texas Tech University's Reese Technology Center Field Site. Texas Tech University Tech. Rep., 28 pp.

Issa, R. I., 1986: Solution of the implicitly discretized fluid flow equations by operator-splitting. J. Comput. Phys., 62, 40-65, https://doi.org/10.1016/0021 -9991(86)90099-9.

Kelley, N. D., 2011: Turbulence-turbine interaction: The basis for the development of the TurbSim stochastic simulator. National Renewable Energy Laboratory Rep. NREL/TP-5000-52353, 332 pp., www.nrel.gov /docs/fy12osti/52353.pdf.

LeMone, M. A., 1976: Modulation of turbulence energy by longitudinal rolls in an unstable planetary boundary layer. J. Atmos. Sci., 33, 1308-1320, https://doi .org/10.1175/1520-0469(1976)033<1308:MOTEBL $>2.0 . \mathrm{CO} ; 2$.

Mahoney, W. P., and Coauthors, 2012: A wind power forecasting system to optimize grid integration. IEEE Trans. Sustainable Energy, 3, 670-682, https://doi .org/10.1109/TSTE.2012.2201758.

Mazzaro, L. J., D. Muñoz-Esparza, J. K. Lundquist, and R. R. Linn, 2017: Nested mesoscale-to-LES modeling of the atmospheric boundary layer in the presence of under-resolved convective structures. J. Adv. Model. Earth Syst., 9, 1795-1810, https://doi .org/10.1002/2017MS000912.

_ , E. Koo, D. Muñoz-Esparza, J. K. Lundquist, and R. R. Linn, 2019: Random force perturbations: A new extension of the cell perturbation method for turbulence generation in multiscale boundary layer simulations. J. Adv. Model. Earth Syst., 11, 2311-2329, https://doi.org/10.1029/2019MS001608.
Mirocha, J. D., J. K. Lundquist, and B. Kosović, 2010: Improved large-eddy simulation subfilter turbulence models in the Advanced Research WRF version 3. Mon. Wea. Rev., 138, 4212-4228, https://doi .org/10.1175/2010MWR3286.1.

—, B. Kosović, and G. Kirkil, 2014: Resolved turbulence characteristics in large-eddy simulations nested within mesoscale simulations using the Weather Research and Forecasting Model. Mon. Wea. Rev., 142, 806-831, https://doi.org/10.1175 /MWR-D-13-00064.1.

—, D. A. Rajewski, N. Marjanovic, J. K. Lundquist, B. Kosović, C. Draxl, and M. J. Churchfield, 2015: Investigating wind turbine impacts on near-wake flow using profiling lidar data and large-eddy simulations with an actuator disk model. J. Renewable Sustainable Energy, 7, 043143, https://doi.org/10.1063 $/ 1.4928873$.

— sensitivities to variations of configuration and forcing parameters in canonical boundary layer flows for wind energy applications. Wind Energy Sci., 3, 589-613, https://doi.org/10.5194/wes-3-589-2018.

Moeng, C.-H., J. Dudhia, J. Klemp, and P. Sullivan, 2007: Examining two-way grid nesting for large-eddy simulation of the PBL using the WRF Model. Mon. Wea. Rev., 135, 2295-2311, https://doi.org/10.1175 /MWR3406.1.

Monin, A. S., and A. M. Obukhov, 1954: Basic laws of turbulent mixing in the surface layer of the atmosphere. Tr. Geofiz. Inst., Akad. Nauk. SSSR, 24, 163-187.

Muñoz-Esparza, D., and B. Kosović, 2018: Generation of inflow turbulence in large-eddy simulations of nonneutral atmospheric boundary layers with the cell perturbation method. Mon. Wea. Rev., 146, 18891909, https://doi.org/10.1175/MWR-D-18-0077.1.

,$- \ldots$, J. D. Mirocha, and J. van Beeck, 2014: Bridging the transition from mesoscale to microscale turbulence in numerical weather prediction models. Bound.-Layer Meteor., 153, 409-440, https://doi .org/10.1007/s10546-014-9956-9.

$-, \ldots, \ldots$, and J. D. Mirocha, 2015: A stochastic perturbation method to generate inflow turbulence in large-eddy simulation models: Application to neutrally stratified atmospheric boundary layers. Phys. Fluids, 27, 035102, https://doi.org/10.1063 /1.4913572.

—, J. K. Lundquist, J. A. Sauer, B. Kosović, and R. R. Linn, 2017: Coupled mesoscale-LES modeling of a diurnal cycle during the CWEX-13 field campaign: From weather to boundary-layer eddies. $J$. Adv. Model. Earth Syst., 9, 1572-1594, https://doi .org/10.1002/2017MS000960. 
National Renewable Energy Laboratory, 2015: SOWFA. National Renewable Energy Laboratory, https://nwtc .nrel.gov/SOWFA.

- 2018: Exawind project demonstrates bladeresolved simulation of the NREL $5 \mathrm{MW}$ reference wind turbine. U.S. Department of Energy Office of Science and National Nuclear Security Administration, www.exascaleproject.org/exawind-project -demonstrates-blade-resolved-simulation-of-the -nrel-5-mw-reference-wind-turbine/.

Olson, J. B., and Coauthors, 2019: Improving wind energy forecasting through numerical weather prediction model development. Bull. Amer. Meteor. Soc., https://doi.org/10.1175/BAMS-D-18-0040.1, in press.

OpenFOAM, 2014: The open source CFD toolbox, version 2.4.x. OpenCFD Limited, accessed November 2018, www.openfoam.com.

Quon, E. W., A. S. Ghate, and S. K. Lele, 2018: Enrichment methods for inflow turbulence generation in the atmospheric boundary layer. J. Phys. Conf. Ser., 1037, 072054, https://doi.org/10.1088/1742 $-6596 / 1037 / 7 / 072054$.

Rai, R. K., L. K. Berg, B. Kosović, J. D. Mirocha, M. S. Pekour, and W. J. Shaw, 2017: Comparison of measured and numerically simulated turbulence statistics in a convective boundary layer over complex terrain. Bound.-Layer Meteor., 163, 69-89, https:// doi.org/10.1007/s10546-016-0217-y.

,,--- , S. E. Haupt, J. D. Mirocha, B. Ennis, and C. Draxl, 2019: Evaluation of the impact of horizontal grid spacing in terra incognita on coupled mesoscale-microscale simulations using the WRF framework. Mon. Wea. Rev., 147, 1007-1027, https:// doi.org/10.1175/MWR-D-18-0282.1.

Rajewski, D. A., E. S. Takle, J. K. Lundquist, J. H. Prueger, R. L. Pfeiffer, J. L. Hatfield, K. K. Spoth, and R. K. Doorenbos, 2014: Changes in fluxes of heat, $\mathrm{H}_{2} \mathrm{O}$, and $\mathrm{CO}_{2}$ caused by a large wind farm. Agric. For. Meteor., 194, 175-187, https://doi.org/10.1016/j .agrformet.2014.03.023.

Rodrigo, J. S., M. Churchfield, and B. Kosovic, 2016: Atmospheric boundary layer modeling based on mesoscale tendencies and data assimilation at microscale. Wind Energy Sci., 2, 35-54, https://doi.org /10.5194/wes-2-35-2017.

Schumann, U., 1975: Subgrid scale model for finite difference simulations of turbulent flows in plane channels and annuli. J. Comput. Phys., 18, 376-404, https://doi.org/10.1016/0021-9991(75)90093-5.

Shaw, W., and Coauthors, 2019: The Second Wind Forecast Improvement Project (WFIP2): General overview. Bull. Amer. Meteor. Soc., 100, 1687-1699, https://doi .org/10.1175/BAMS-D-18-0036.1.
Skamarock, W., and Coauthors, 2008: A description of the Advanced Research WRF version 3. NCAR Tech. Note NCAR/TN-475+STR, 113 pp., https://doi .org/10.5065/D68S4MVH.

Sorbjan, Z., 2004: Large-eddy simulations of the baroclinic mixed layer. Bound.-Layer Meteor., 112, 57-80, https://doi.org/10.1023/B:BOUN.0000020161.99887.b3.

Sørensen, J. N., and W. Z. Shen, 2002: Numerical modeling of wind turbine wakes. J. Fluids Eng., 124, 393-399, https://doi.org/10.1115/1.1471361.

Stensrud, D. J., 2007: Parameterization Schemes: Keys to Understanding Numerical Weather Prediction Models. Cambridge University Press, 478 pp.

Warner, T. T., 2011: Numerical Weather and Climate Prediction. Cambridge University Press, 526 pp.

Weckwerth, T. M., T. W. Horst, and J. W. Wilson, 1999: An observational study of the evolution of horizontal convective rolls. Mon. Wea. Rev., 127, 2160-2179, https://doi.org/10.1175/1520-0493(1999)127<2160:AO SOTE $>2.0 . C O ; 2$.

Weissman, G., R. Sargent, and B. Fanshaw, 2018: Renewables on the rise 2019: A decade of progress toward a clean energy future. Environment America Rep., 46 pp., https://environmentamerica.org/sites /environment/files/reports/AME_Renewables-on -the-Rise_Jul18-Web.pdf.

Wilczak, J. M., and Coauthors, 2019: The Second Wind Forecast Improvement Project (WFIP2): Observational field campaign. Bull. Amer. Meteor. Soc., 100, 1701-1723, https://doi.org/10.1175/BAMS -D-18-0035.1.

Wyngaard, J. C., 2004: Toward numerical modeling in the "terra incognita." J. Atmos. Sci., 61, 1816-1826, https://doi.org/10.1175/1520-0469(2004)061<1816:TN MITT>2.0.CO;2.

Yang, B., and Coauthors, 2017: Sensitivity of turbineheight wind speeds to parameters in planetary boundary-layer and surface-layer schemes in the Weather Research and Forecasting Model. Bound.Layer Meteor., 162, 117-142, https://doi.org/10.1007 /s10546-016-0185-2.

Yang, Q., L. K. Berg, M. Pekour, J. D. Fast, R. K. Newsom, M. Stoelinga, and C. Finley, 2013: Evaluation of WRF-predicted near-hub-height winds and ramp events over a Pacific Northwest site with complex terrain. J. Appl. Meteor. Climatol., 52, 1753-1763, https://doi.org/10.1175/JAMC-D-12-0267.1.

Zajaczkowski, F. J., S. E. Haupt, and K. J. Schmehl, 2011: A preliminary study of assimilating numerical weather prediction data into computational fluid dynamics models for wind prediction. J. Wind Eng. Ind. Aerodyn., 99, 320-329, https://doi.org/10.1016/j .jweia.2011.01.023. 


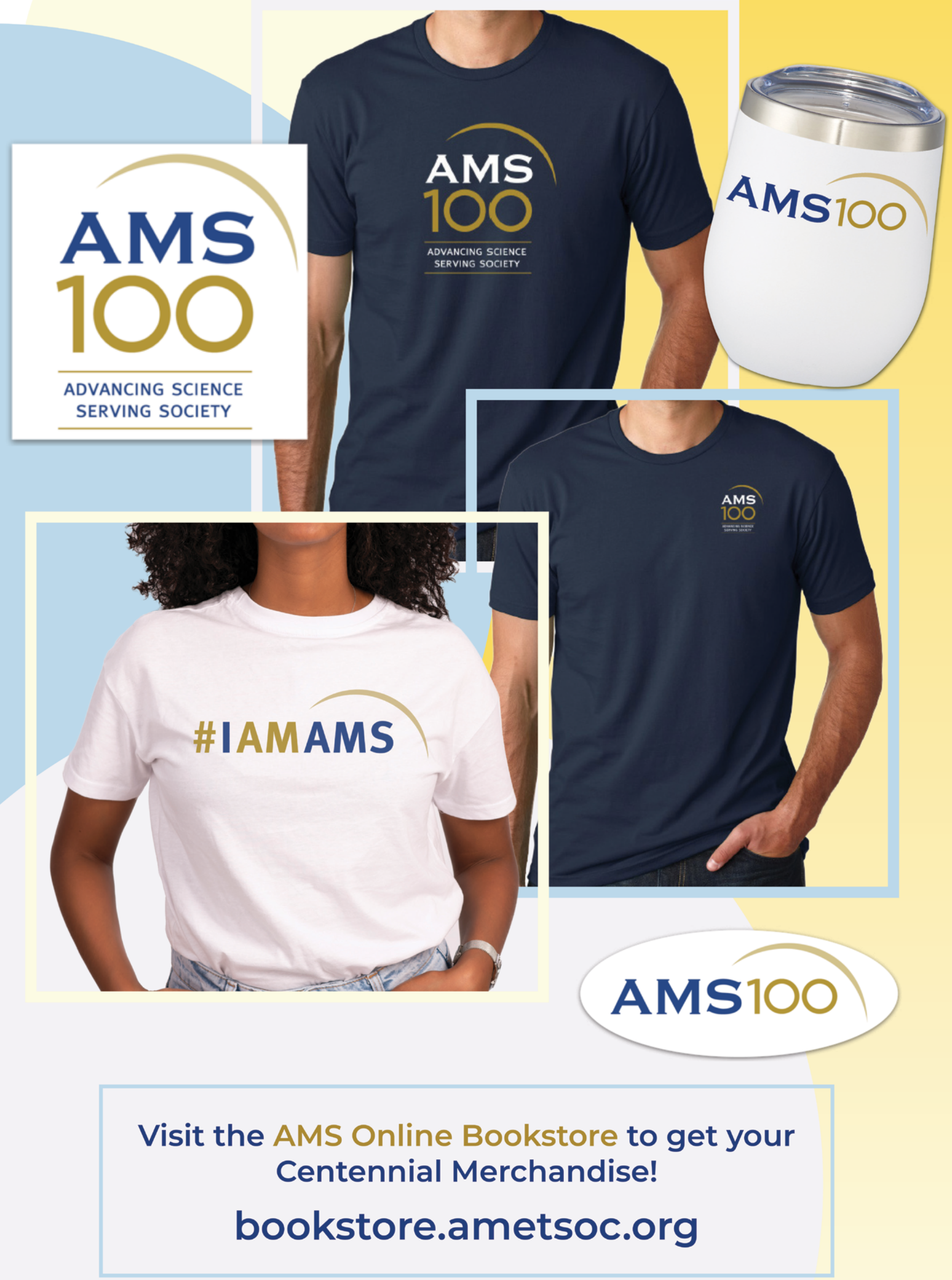

